\title{
CONVERGENCE OF FUNCTIONS: EQUI-SEMICONTINUITY
}

\author{
BY
}

SZYMON DOLECKI, GABRIELLA SALINETTI AND ROGER J.-B. WETS

\begin{abstract}
We study the relationship between various types of convergence for extended real-valued functionals engendered by the associated convergence of their epigraphs; pointwise convergence being treated as a special case. A condition of equi-semicontinuity is introduced and shown to be necessary and sufficient to allow the passage from one type of convergence to another. A number of compactness criteria are obtained for families of semicontinuous functions; in the process we give a new derivation of the Arzelá-Ascoli Theorem.
\end{abstract}

Given a space $X$, by $\bar{R}^{X}$ we denote the space of all functions defined on $X$ and with values in $\bar{R}$, the extended reals. We are interested in the relationship between various notions of convergence in $\bar{R}^{X}$, in particular, but not exclusively, between pointwise convergence and that induced by the convergence of the epigraphs. We extend and refine the results of De Giorgi and Franzoni [1975] (collection of "equi-Lipschitzian" functions with respect to pseudonorms) and of Salinetti and Wets [1977] (sequences of convex functions on reflexive Banach spaces). The range of applicability of the results is substantially enlarged, in particular the removal of the convexity, reflexivity and the norm dependence assumptions is significant in many applications; for illustrative purposes an example is worked out further on. The work in this area was motivated by: the search for "valid" approximations to extremal statistical problems, variational inequalities and difficult optimization problems, cf. the above mentioned articles. Because we rely only on minimal properties for the topology of the domain space and for the class of functions involved, the derivation itself takes on an elementary and insightful character.

By their nature, the results are asymmetric; semicontinuity is a one-sided concept. We have chosen to deal with lower semicontinuity and epigraphs rather than upper semicontinuity and hypographs. Every assertion in one setting has its obvious counterpart in the other. This choice however, does condition the addition rule for the extended reals, viz. $(+\infty)+a=+\infty$ for all $a \in \bar{R}$ and $(-\infty)+a=-\infty$ for all $a \in[-\infty,+\infty[$. Also, note that we are working with the extended reals, thus every collection of elements of $\bar{R}$ has lower and upper bounds in $\bar{R}$; all limits involving extended-real numbers must be interpreted in that sense.

Received by the editors December 28, 1981 and, in revised form, March 25, 1982. 1980 Mathematics Subject Classification. Primary 49D99, 58E30; Secondary 54A20. 
I. Limit functions. Let $(X, \tau)$ be a topological space and $f$ a generic element of $\bar{R}^{X}$. The effective domain of $f$ is

$$
\operatorname{dom} f=\{x \in X \mid f(x)<+\infty\}
$$

and its epigraph is

$$
\text { epi } f=\{(x, \eta) \in X \times R \mid f(x) \leqslant \eta\} .
$$

The function $f: X \rightarrow \bar{R}$ is $\tau$-lower semicontinuous $\left(\tau\right.$-l.sc.) at $x$, if there exists $\varepsilon_{x}>0$ such that

$\left(\mathrm{d}_{0}\right)$ to every $\left.\varepsilon \in\right] 0, \varepsilon_{x}\left[\right.$ there corresponds $V \in \mathcal{G}_{\tau}(x)$ such that

$$
\inf _{y \in V} f(y) \geqslant \min \left[f(x)-\varepsilon, \varepsilon^{-1}\right]
$$

where $\mathcal{G}_{\tau}(x)$ is the family of (open) $\tau$-neighborhoods of $x$. The function $f$ is $\tau$-lower semicontinuous if the condition holds for every $x \in X$, or equivalently if epi $f$ is closed in $X \times R$ with respect to the product topology of $\tau$ and the natural topology for $R$. If $\sigma \supset \tau$, i.e. $\sigma$ is a topology on $X$ finer than $\tau$, then $f \sigma$-1.sc. implies that $f$ is $\tau$-1.sc.

To define limits of collection of functions, i.e., elements of $\bar{R}^{X}$, we adopt the following framework: $N$ is an index space and $\mathcal{H}$ is a filter on $N$. If $\tau$ has a local countable base at each point, it would be sufficient to consider limits in terms of sequences; unfortunately many interesting functional spaces do not have this property. The $e_{\tau}$-limit inferior of a filtered collection of functions $\left\{f_{\nu}, \nu \in N\right\}$ is denoted by $\mathrm{li}_{\tau} f_{\nu}$, and is defined by

$$
\left(\mathrm{li}_{\tau} f_{\nu}\right)(x)=\sup _{G \in \mathcal{G}_{\tau}(x)} \sup _{H \in \mathcal{H}} \inf _{\nu \in H} \inf _{y \in G} f_{\nu}(y) .
$$

The $e_{\tau}$-limit superior is denoted by $\mathrm{ls}_{\tau} f_{\nu}$, and is defined similarly,

$$
\left(1 \mathrm{~s}_{\tau} f_{\nu}\right)(x)=\sup _{G \in \mathcal{G}_{\tau}(x)} \inf _{H \in \mathcal{H}} \sup _{\nu \in H} \inf _{y \in G} f_{\nu}(y) .
$$

In the literature on $\Gamma$-convergence, these two functions are known as the $\Gamma^{-}(\tau)$-limit inferior and the $\Gamma^{-}(\tau)$-limit superior respectively, cf. De Giorgi and Franzoni [1975]. ${ }^{\prime}$

By $\ddot{\mathscr{H}}$ we denote the grill associated with the filter $\mathcal{H}$, i.e. the family of subsets of $N$ that meet every set $H$ in $\mathcal{K}$. Given any collection $\left\{a_{\nu} \in \bar{R}, \nu \in N\right\}$ it is easy to verify the identity

$$
\sup _{H^{\prime} \in \mathscr{H} \mathcal{C}} \inf _{\nu \in H^{\prime}} a_{\nu}=\inf _{H \in \mathcal{H}} \sup _{\nu \in H} a_{\nu}
$$

\footnotetext{
'When convergence in the $\tau$ topology can be defined in terms of sequential convergence, the limit functions can also be obtained as follows: let $N=\{1,2, \ldots\}$, then

$$
\left(\operatorname{li}_{\tau} f_{\nu}\right)(x)=\inf _{\substack{\left\{\nu_{\mu}\right\} \subset N \\\left\{x_{\mu} \rightarrow x\right\}}} \lim _{\mu \in N} \inf f_{\nu_{\mu}}\left(x_{\mu}\right)
$$

and $\left(\operatorname{ls}_{\tau} f_{\nu}\right)(x)=\inf _{\left\{x_{\nu} \rightarrow x\right\}} \lim _{\nu} \sup f_{\nu}\left(x_{\nu}\right)$, where in the first expression the infimum is over all subsequences of functions $\left\{f_{\mu_{\nu}}, \mu \in N\right\}$ and all sequences $\left\{x_{\mu}, \mu \in N\right\}$ converging to $x$.
} 
if we use the fact that $\mathcal{H}$ is the "grill" of $\ddot{\mathcal{H}}$, i.e. the collection of subsets of $N$ that meet every set in $\ddot{\mathcal{H}}$. From this it follows that

$$
\left(1 \mathrm{~s}_{\tau} f_{\nu}\right)(x)=\sup _{G \in \mathcal{G}_{\tau}(x)} \sup _{H \in \ddot{\mathscr{K} C}} \inf _{\nu \in H} \inf _{y \in G} f_{\nu}(y) .
$$

Since $\mathscr{H} \subset \ddot{H}$, clearly

$$
\operatorname{li}_{\tau} f_{\nu} \leqslant \operatorname{ls}_{\tau} f_{\nu} .
$$

The collection $\left\{f_{\nu}, \nu \in N\right\}$ admits an $e_{\tau}$-limit, denoted by $\operatorname{lm}_{\tau} f_{\nu}$, if

$$
\operatorname{ls}_{\tau} f_{\nu}=\operatorname{li}_{\tau} f_{\nu}=\operatorname{lm}_{\tau} f_{\nu},
$$

in which case the $f_{\nu}$ are said to epi-converge to $\operatorname{lm}_{\tau} f_{\nu}$. This terminology comes from the fact that epi $\operatorname{lm}_{\tau} f_{\nu}$ is the limit of the epigraphs of the $f_{\nu}$; this is made explicit further on.

The limit inferior Li $C_{\nu}$ and limit superior $L s C_{\nu}$ of a filtered collection $\left\{C_{\nu}, \nu \in N\right\}$ of subsets of a topological space are defined by

$$
\operatorname{Li} C_{\nu}=\bigcap_{H \in \ddot{\mathcal{H}}} \operatorname{cl}\left(\bigcup_{\nu \in H} C_{\nu}\right)
$$

and

$$
L s C_{\nu}=\bigcap_{H \in \mathcal{H}} \operatorname{cl}\left(\bigcup_{\nu \in H} C_{\nu}\right)
$$

Since $\mathcal{H} \subset \ddot{H}$,

$$
\text { Li } C_{\nu} \subset \operatorname{Ls} C_{\nu} \text {. }
$$

The filtered collection $\left\{C_{\nu}, \nu \in N\right\}$ is said to have a limit, $\operatorname{Lm} C_{\nu}$, if the limits inferior and superior coincide, i.e.,

$$
\text { Ls } C_{\nu}=\operatorname{Lm} C_{\nu}=\operatorname{Li} C_{\nu} \text {. }
$$

All these limit sets are closed as follows directly from the definitions.

Proposition 1.9 (Mosco [1969]). Suppose that $\left\{f_{\nu}, \nu \in N\right\} \subset \bar{R}^{X}$ is a filtered collection of functions. Then

$$
\text { epi } \operatorname{li}_{\tau} f_{\nu}=L s \text { epi } f_{\nu}
$$

and

$$
\text { epi } \operatorname{ls}_{\tau} f_{\nu}=L i \text { epi } f_{\nu}
$$

where limits in $X \times R$ are taken with respect to the product topology of $\tau$ and the natural topology for $R$.

Proof. We first derive (1.10). From the definition (1.6) of $L s$ epi $f_{\nu}$, it follows that $(x, \alpha) \in L s$ epi $f_{\nu}$ if and only if

$$
(x, \alpha) \in \operatorname{cl}\left(\bigcup_{\nu \in H} \text { epi } f_{\nu}\right) \quad \text { for all } H \in \mathcal{H}
$$


or equivalently - because the sets involved are epigraphs - if and only if for all $\varepsilon>0$ and $G \in \mathcal{G}_{\tau}(x)$ such that

$$
G \times]-\infty, \alpha+\varepsilon\left[\cap\left(\bigcup_{\nu \in H} \text { epi } f_{\nu}\right) \neq \varnothing \quad \text { for all } H \in \mathcal{H}\right.
$$

or still, if and only if for every $H \in \mathcal{H}, \varepsilon>0$ and $G \in \mathcal{G}_{\tau}(x)$ there correspond $\nu \in H$ and $y \in G$ such that $\alpha+\varepsilon \geqslant f_{\nu}(y)$. This holds, if and only if

$$
\alpha \geqslant \sup _{G \in \mathcal{G}_{\tau}(x)} \sup _{H \in \mathscr{K}} \inf _{\nu \in H} \inf _{y \in G} f_{\nu}(y)
$$

and, as follows from (1.1), if and only if $\alpha \geqslant\left(\mathrm{li}_{\tau} f_{\nu}\right)(x)$ or equivalently, if and only if $(x, \alpha) \in \operatorname{epi~li}_{\tau} f_{\nu}$.

In view of (1.4), the proof of (1.11) follows from exactly the same argument with the grill $\ddot{\mathcal{H}}$ replacing $\mathcal{H}$.

COROLlaRY 1.12. Given any filtered collection of functions $\left\{f_{\nu}, \nu \in N\right\} \subset \bar{R}^{X}$, the functions $\operatorname{li}_{\tau} f_{\nu}, \mathrm{ls}_{\tau} f_{\nu}$, and $\operatorname{lm}_{\tau} f_{\nu}$ if it exists, are $\tau$-lower semicontinuous.

Proof. The lower semicontinuity follows directly from (1.10) and (1.11) since they imply that the epigraphs are closed.

We shall be interested in the implications of a change in topology for $X$. In particular, we have the following

Proposition 1.13. Suppose that $\sigma$ and $\tau$ are two topologies defined on $X$ such that $\sigma \supset \tau$. Then

$$
\operatorname{li}_{\tau} f_{\nu} \leqslant \mathrm{li}_{\sigma} f_{\nu},
$$

and

$$
1 \mathrm{~s}_{\tau} f_{\nu} \leqslant 1 \mathrm{~s}_{\sigma} f_{\nu} .
$$

Proof. This follows from the definitions (1.1) and (1.2) and the fact that $\sigma \supset \tau$ implies that $\mathcal{G}_{\sigma}(x) \supset \mathcal{G}_{\tau}(x)$.

In some applications, in particular those involving variational inequalities, it is useful to use a stronger notion of limit function. Again, let $\sigma$ and $\tau$ be two topologies defined on $X$, the $e_{\tau, \sigma}$-limit of a collection of functions $\left\{f_{\nu}, \nu \in N\right\} \subset \bar{R}^{X}$, denoted by $\operatorname{lm}_{\tau, \sigma} f_{\nu}$, exists if

$$
\operatorname{li}_{\tau} f_{\nu}=\operatorname{lm}_{\tau, \sigma} f_{\nu}=\operatorname{ls}_{\sigma} f_{\nu} .
$$

The case of interest is $\sigma \supset \tau$, this models the situation when $X$ is a normed linear (functional) space, and $\sigma$ and $\tau$ are respectively the strong and weak topologies; in this setting this limit function is called the Mosco limit, cf. Mosco [1969] and Attouch [1979a].

Proposition 1.17. Suppose that $\sigma$ and $\tau$ are two topologies defined on $X$ such that $\sigma \supset \tau$. Moreover, suppose that $\operatorname{lm}_{\tau, \sigma} f_{\nu}$ exists. Then

$$
\operatorname{lm}_{\sigma} f_{\nu}=\operatorname{lm}_{\tau, \sigma} f_{\nu}=\operatorname{lm}_{\tau} f_{\nu} .
$$

Proof. This follows directly from Proposition 1.13, inequality (1.5) and the definition (1.16) of $\operatorname{lm}_{\tau, \sigma} f_{\nu}$. 
II. $\tau / \sigma$-equi-semicontinuity. As already indicated in $\S \mathrm{I}$, we are interested in exploring the relationship between the limit functions of a collection of functions $\left\{f_{\nu}, \nu \in N\right\} \subset \bar{R}^{X}$, when $X$ is equipped with different topologies, say $\sigma$ and $\tau$. Recall that for variational problems epi-convergence essentially implies the convergence of the solutions. It is thus useful to have conditions that allow us to pass from epi-convergence in a given topology to epi-convergence in a finer topology because it would yield stronger continuity properties for the solution of the limit problem, consult Example 2.11 below; see also Attouch [1979b]. Finally, a special and extreme case is when $\sigma=\imath$, the discrete topology. The study of the connections between $\operatorname{lm}_{\tau}$ and $1 \mathrm{~m}$, becomes that of the relationship between epi-convergence and pointwiseconvergence. This is particularly useful in the design of approximation schemes for optimization problems. We deal with this special case of pointwise-convergence at the end of this section.

We shall see that when $\sigma \supset \tau$, the inequalities $\operatorname{li}_{\tau} f_{\nu} \leqslant \operatorname{li}_{\sigma} f_{\nu}(1.14)$ and $\operatorname{ls}_{\tau} f_{\nu} \leqslant \operatorname{ls}_{\sigma} f_{\nu}$ (1.15), relating the $e_{\tau}$-limits inferior and superior, become equalities if the family of functions $\left\{f_{\nu}, \nu \in N\right\}$ is $\tau / \sigma$-equi-lower semicontinuous. This property is not only sufficient (Theorem 2.3) but also necessary (Theorem 2.9). It is a sort of "local" compactness condition; this is clarified in $\$ I V .^{2}$

Definition 2.1. A filtered collection of functions $\left\{f_{\nu}, \nu \in N\right\} \subset \bar{R}^{X}$ is $\tau / \sigma$-equi-lower semicontinuous at $x(\tau / \sigma$-equi-l.sc. at $x)$ if there exists $\varepsilon_{x}>0$ such that

(d) to every $\varepsilon \in] 0, \varepsilon_{x}\left[\right.$ and every $W \in \mathcal{G}_{\sigma}(x)$ there correspond $H \in \mathcal{H}$ and $V \in \mathcal{G}_{\tau}(x)$ such that for all $\nu \in H$

$$
\inf _{y \in V} f_{\nu}(y) \geqslant \min \left[\inf _{y \in W} f_{\nu}(y)-\varepsilon, \varepsilon^{-1}\right] .
$$

The collection is $\tau / \sigma$-equi-l.sc. if (d) holds for every $x \in X$.

If $\sigma \subset \tau$, then (d) is trivially satisfied, simply take $V=W$ and $H$ arbitrary, and thus in this situation any collection of functions is $\tau / \sigma$-equi-l.sc. The only case of genuine interest in applications, as far as we can tell, is when $\tau$ is coarser than $\sigma$, however the results derived here do not involve any such restrictions on the topologies.

Proposition 2.2. Suppose that $\sigma_{2} \supset \sigma_{1}$ and $\tau_{2} \subset \tau_{1}$. Then for any collection of functions, $\tau_{2} / \sigma_{2}$-equi-lower semicontinuity implies $\tau_{1} / \sigma_{1}$-equi-lower semicontinuity.

Proof. Simply use Definition 2.1, the inclusions $\mathcal{G}_{\sigma_{2}}(x) \supset \mathcal{G}_{\sigma_{1}}(x)$ and $\mathcal{G}_{\tau_{2}}(x) \subset$ $\mathcal{G}_{\tau_{1}}(x)$.

THEOREM 2.3. Suppose the filtered collection of functions $\left\{f_{\nu}, \nu \in N\right\} \subset \bar{R}^{X}$ is $\tau /$ o-equi-l.sc. Then

$$
\operatorname{li}_{\sigma} f_{\nu} \leqslant \operatorname{li}_{\tau} f_{\nu}
$$

\footnotetext{
${ }^{2}$ A function $f$ from $X$ to $\bar{R}$ is $\tau / \sigma-l . s c$. if (d) holds and $f_{\nu}=f$ for all $\nu \in N$. If $\tau \supset \sigma$ the concept is essentially meaningless since then any function $f \in \bar{R}^{X}$ is then $\tau / \sigma-1 . s c$. If $\sigma \supset \tau$, then $f$ is $\tau / \sigma$-l.sc. if and only if $\tau$-cl $(\sigma$-cl epi $f)=\sigma$-cl epi $f$. In particular if $\sigma=\iota$ then $\tau / \iota$-l.sc. corresponds to the usual notion of $\tau$-1.sc.
} 
and

$$
1 \mathrm{~s}_{\sigma} f_{\nu} \leqslant 1 \mathrm{~s}_{\tau} f_{\nu} .
$$

Proof. We start by showing that the $\tau / \sigma$-equi-lower semicontinuity conditions imply that

$$
\operatorname{dom} \operatorname{li}_{\tau} f_{\nu} \subset \operatorname{dom~li}_{\sigma} f_{\nu} .
$$

Suppose $x \notin \operatorname{dom~li}_{\sigma} f_{\nu}$, i.e. $\operatorname{li}_{\sigma} f_{\nu}(x)=\infty$. From the definition (1.1) of $\operatorname{li}_{\sigma} f_{\nu}$ it follows that given any $\varepsilon>0$ sufficiently small, we can find $G_{\varepsilon} \in \mathcal{G}_{\sigma}(x)$ and $H_{\varepsilon} \in \mathcal{H}$ such that for all $\nu$ in $H_{\varepsilon}$,

$$
\inf _{y \in G_{\varepsilon}} f_{\nu}(y) \geqslant \varepsilon^{-1}-\varepsilon
$$

which implies that for all $\nu \in H_{\varepsilon}$,

$$
\min \left[\inf _{y \in G_{\varepsilon}} f_{\nu}(y)-\varepsilon, \varepsilon^{-1}\right]=\varepsilon^{-1} .
$$

Since $\left\{f_{\nu}, \nu \in N\right\}$ is $\tau / \sigma$-equi-1.sc., it follows that for $\varepsilon>0$ sufficiently small and $G_{\varepsilon} \in \mathcal{G}_{\sigma}(x)$ there correspond $V \in \mathcal{G}_{\tau}(x)$ and $H^{\prime} \in \mathcal{H}$ such that for all $\nu \in H^{\prime}$,

$$
\inf _{y \in V} f_{\nu}(y) \geqslant \min \left[\inf _{y \in G_{\varepsilon}} f_{\nu}(y)-\varepsilon, \varepsilon^{-1}\right]
$$

which combined with the preceding relation, yields

$$
\inf _{y \in V} f_{\nu}(y) \geqslant \varepsilon^{-1}
$$

for all $\nu \in H_{\varepsilon} \cap H^{\prime}(\in \mathcal{H})$. We now simply use the definition (1.1) of $\operatorname{li}_{\tau} f_{\nu}$ to conclude that $\left(\operatorname{li}_{\tau} f_{\nu}\right)(x) \geqslant \varepsilon^{-1}$. Since this holds for arbitrarily small $\varepsilon>0$, it follows that $\left(\mathrm{li}_{\tau} f_{\nu}\right)(x)=\infty$, and thus $x \notin \operatorname{dom~li}_{\tau} \mathrm{f}_{\nu}$.

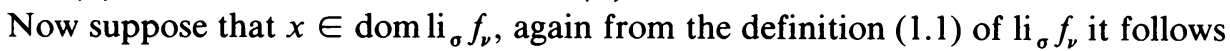
that there exists $G_{\varepsilon} \in \mathcal{G}_{\sigma}(x)$ and $H_{\varepsilon} \in \mathcal{H}$ such that for all $\nu \in H_{\varepsilon}$,

$$
\left(\operatorname{li}_{\sigma} f_{\nu}\right)(x) \leqslant\left[\inf _{y \in G_{\varepsilon}} f_{\nu}(y)\right]+\varepsilon
$$

In turn (d) guarantees the existence of $V \in \mathcal{G}_{\tau}(x)$ and $H^{\prime} \in \mathcal{K}$ such that for all $\nu \in H^{\prime}$,

$$
\inf _{y \in G_{\varepsilon}} f_{\nu}(y) \leqslant \inf _{y \in V} f_{\nu}(y)+\varepsilon
$$

and hence for all $\nu \in H_{\varepsilon} \cap H^{\prime}(\in \mathcal{H})$, we have

$$
\left(\operatorname{li}_{\sigma} f_{\nu}\right)(x) \leqslant \inf _{\nu \in H_{\varepsilon} \cap H^{\prime}} \inf _{y \in V} f_{\nu}(y)+\varepsilon .
$$

But this implies that $\left(\operatorname{li}_{\sigma} f_{\nu}\right)(x) \leqslant\left(\operatorname{li}_{\tau} f_{\nu}\right)(x)+2 \varepsilon$, cf. (1.1). And since this holds for every $\varepsilon>0$ sufficiently small, it follows that $\left(\operatorname{li}_{\sigma} f_{\nu}\right)(x) \leqslant\left(\operatorname{li}_{\tau} f_{\nu}\right)(x)$ for every $x \in$ dom $\operatorname{li}_{\sigma} f_{\nu}$. This combined with dom $\operatorname{li}_{\tau} f_{\nu} \subset \operatorname{dom~li}_{\sigma} f_{\nu}$ yields (2.4).

The proof of $(2.5)$ follows the same lines, replacing li by ls, $\mathcal{H}$ by $\ddot{\mathscr{H}}$, recalling that $\mathcal{H} \subset \ddot{\mathcal{H}}$ and relying on (1.4) rather than (1.1). 
Corollary 2.6. Convergence Theorem. Suppose $\sigma \supset \tau$ and that the filtered collection of functions $\left\{f_{\nu}, \nu \in N\right\} \subset \bar{R}^{X}$ is $\tau / \sigma$-equi-l.sc. Then

$$
\operatorname{li}_{\sigma} f_{\nu}=\operatorname{li}_{\tau} f_{\nu},
$$

and

$$
\operatorname{ls}_{\boldsymbol{\sigma}} f_{\nu}=1 \mathrm{~s}_{\tau} f_{\nu} .
$$

Also, $f=\operatorname{lm}_{\sigma} f_{\nu}$ if and only if $f=\operatorname{lm}_{\tau} f_{\nu}$.

Proof. The identities (2.7) and (2.8) follow directly from the theorem and Proposition 1.13. When combined with the definition of $\operatorname{lm}_{\sigma}\left(\right.$ or $\left.\operatorname{lm}_{\tau}\right)$ they yield the remainder.

We next show that $\tau / \sigma$-equi-semicontinuity is a minimal condition which allows us to pass from the epi-convergence in one topology to the epi-convergence in another topology.

THEOREM 2.9. Suppose that $\left\{f_{\nu}, \nu \in N\right\} \subset \bar{R}^{X}$ is a filtered collection of functions such that $-\infty<\operatorname{ls}_{\sigma} f_{\nu} \leqslant \operatorname{li}_{\tau} f_{\nu}$. Then the collection $\left\{f_{\nu}, \nu \in N\right\}$ is $\tau / \sigma$-equi-l.sc. Moreover if $\sigma \supset \tau$, then also

$$
\operatorname{lm}_{\sigma} f_{\nu}=\operatorname{lm}_{\tau} f_{\nu} .
$$

Proof. The equality (2.10) follows immediately from the hypotheses, (1.5) and Proposition 1.13. We now establish the $\tau / \sigma$-equi-lower semicontinuity. To say that (d) fails at $x$, means that given any $\varepsilon_{x}>0$, we can find $\left.\varepsilon \in\right] 0, \varepsilon_{x}\left[\right.$ and $G \in \mathcal{G}_{\sigma}(x)$ such that for any pair $H \in \mathcal{H}$ and $V \in \mathcal{G}_{\tau}(x)$, we have that

$$
\inf _{\nu \in H} \inf _{y \in V} f_{\nu}(y)<\min \left[\inf _{y \in G_{\varepsilon}} f_{\nu}(y)-\varepsilon, \varepsilon^{-1}\right]
$$

which implies that

$$
\operatorname{li}_{\tau} f_{\nu}(x) \leqslant \varepsilon^{-1} .
$$

This means that if (d) fails it necessarily fails for some $x \in \operatorname{dom} \mathrm{li}_{\tau} f_{\nu}$, in fact for some $x$ at which $\operatorname{li}_{\tau} f_{\nu}$ is finite since by assumption $-\infty<\mathrm{li}_{\tau} f_{\nu}$.

Thus let us suppose that (d) fails at $x$ with $-\infty<\operatorname{ls}_{\sigma} f_{\nu}(x) \leqslant \operatorname{li}_{\tau} f_{\nu}(x)<\infty$. It means that for any $\varepsilon_{x}>0$, there exists $\varepsilon>0$ and $W \in \mathcal{G}_{\sigma}(x)$ such that for every $H \in \mathcal{H}$ and $V \in \mathcal{G}_{\tau}(x)$,

$$
\varepsilon+\inf _{y \in V} f_{\nu}(y)<\inf _{y \in W} f_{\nu}(y)
$$

for some $\nu \in H$. In particular this must hold for some $\nu^{\prime} \in H^{\prime}$ with the pair $\left(H^{\prime}, V_{\varepsilon}\right)$ constructed as follows: from the definitions of $\mathrm{li}_{\tau}(1.1)$ and $\mathrm{ls}_{\sigma}(1.2)$, we get that

(i) there exists $V_{\varepsilon} \in \mathcal{G}_{\tau}(x)$ and $H_{\varepsilon} \in \mathcal{H}$ such that

$$
\operatorname{li}_{\tau} f_{\nu}(x)-\frac{\varepsilon}{4} \leqslant \inf _{\nu \in H_{\varepsilon}} \inf _{y \in V_{f}} f_{\nu}(y) ;
$$

(ii) to $W \in \mathcal{G}_{\sigma}(x)$, there corresponds $H_{W} \in \mathcal{H}$ such that

$$
\operatorname{ls}_{\sigma} f_{\nu}(x)+\frac{\varepsilon}{4} \geqslant \sup _{\nu \in H_{W}} \inf _{\nu \in W} f_{\nu}(y) .
$$


Now define $H_{\varepsilon} \cap H_{W}=H^{\prime}(\in \mathcal{H})$. Because (d) fails for some $\nu^{\prime} \in H^{\prime}$, we have

$$
\varepsilon+\inf _{y \in V_{\varepsilon}} f_{\nu^{\prime}}(y)<\inf _{y \in W} f_{\nu^{\prime}}(y)
$$

and thus also

$$
\varepsilon+\inf _{\nu \in H^{\prime}} \inf _{y \in V_{\varepsilon}} f_{\nu}(y)<\sup _{\nu \in H^{\prime}} \inf _{y \in W} f_{\nu}(y) .
$$

Because $\mathrm{li}_{\tau} f_{\nu}$ is finite, this yields

$$
\begin{aligned}
\operatorname{li}_{\tau} f_{\nu}(x)+\frac{3 \varepsilon}{4} & =\varepsilon+\operatorname{li}_{\tau} f_{\nu}(x)-\frac{\varepsilon}{4} \leqslant \varepsilon+\inf _{\nu \in H_{\varepsilon}} \inf _{y \in V_{\varepsilon}} f_{\nu}(y) \\
& \leqslant \varepsilon+\inf _{\nu \in H^{\prime}} \inf _{y \in V_{\varepsilon}} f_{\nu}(y)<\sup _{\nu \in H^{\prime}} \inf _{y \in W} f_{\nu}(y) \\
& \leqslant \sup _{\nu \in H_{W}} \inf _{y \in W} f_{\nu}(y) \leqslant \operatorname{ls}_{\sigma} f_{\nu}(x)+\frac{\varepsilon}{4} \leqslant \operatorname{li}_{\tau} f_{\nu}(x)+\frac{\varepsilon}{4},
\end{aligned}
$$

or in other words $\mathrm{li}_{\tau} f_{\nu}(x)+\varepsilon / 2<\mathrm{li}_{\tau} f_{\nu}(x)$, a clear contradiction. Thus (d) holds at every $x \in X$ and hence the collection is $\tau / \sigma$-equi-l.sc.

To illustrate the concept of $\tau / \sigma$-equi-l.sc., and its use in the calculus of variations, we include here an example provided by $\mathrm{H}$. Attouch (Paris-Orsay).

EXAMPLE 2.11. Let $\mathcal{F}$ be the following family of functions:

$$
\mathscr{F}=\left\{f \mid f(u)=\int_{\Omega} j(x, D u(x)) d x, u \in H^{1}(\Omega)\right\},
$$

where $(x, z) \mapsto j(x, z): \Omega \times R^{n} \rightarrow[0, \infty[$ is such that

$$
\begin{gathered}
x \mapsto j(x, z) \quad \text { is measurable, } \\
z \mapsto j(x, z) \quad \text { is convex and continuous, } \\
0 \leqslant j(x, z) \leqslant M\left(1+|z|^{2}\right)
\end{gathered}
$$

for some (positive) constant $M$. Then $\mathscr{F}$ is $\mathcal{L}^{\prime}(\Omega) / \mathscr{L}^{\infty}(\Omega)$-equi-lower semicontinuous.

Proof. We have to prove that for any $u \in H^{1}(\Omega)$, given any $\varepsilon>0$ and $\eta>0$, we can find $\rho(\eta)$ such that for all $f \in \mathscr{F}$,

$$
f(v) \geqslant \inf _{w \in B_{\infty}(u, \eta)} f(w)-\varepsilon
$$

for all $v \in B_{1}(u, \rho(\eta))$, where $B_{p}(a, \alpha)$ is the closed ball of $\mathcal{L}^{p}$ of center $a$ and radius $\alpha$. There is no need to consider the term $\varepsilon^{-1}$ which appears in (d) because $u \in H^{1}$ and $j(x, z) \leqslant M\left(1+|z|^{2}\right)$. For the sake of the argument assume that for some $u$ the collection $\mathscr{F}$ is not equi-1.sc., i.e. there exist $\varepsilon>0, \eta>0$ such that for all $\rho>0$, there exist $f_{\rho} \in \mathscr{F}$ and $v_{\rho} \in B_{1}(u, \rho)$ with

$$
f_{\rho}\left(v_{\rho}\right)<\inf _{w \in B_{\infty}(u, \eta)} f_{\rho}(w)-\varepsilon .
$$

As $\rho$ goes to 0 , the $v_{\rho}$ converge to $u$ with respect to the $L^{1}$-norm, hence also in measure. Thus there exists a sequence of positive real numbers $\left(\alpha_{\rho}\right)_{\rho>0}$ such that $\alpha_{\rho} \downarrow 0$ as $\rho$ goes to 0 and

$$
\operatorname{meas}\left[x \in \Omega \| v_{\rho}(x)-u(x) \mid>\alpha_{\rho}\right]<\alpha_{\rho} .
$$


For any $\alpha>0$, let us define $T_{\alpha}$, the contraction map from $R$ into $R$, by

$$
T_{\alpha}(a)=\left[\begin{array}{ll}
a & \text { if }|a| \leqslant \alpha \\
(\operatorname{sgn} a) \alpha & \text { otherwise. }
\end{array}\right.
$$

Now let us consider the sequence $u_{\rho}=u+T_{\alpha_{\rho}}\left(v_{\rho}-u\right)$. Note that $\left\|u_{\rho}-u\right\|_{\infty}=$ $\left\|T_{\alpha_{\rho}}\left(v_{\rho}-u\right)\right\|_{\infty} \leqslant \alpha_{\rho} \rightarrow 0$ as $\rho \rightarrow 0$. On the other hand,

$$
\begin{aligned}
D u_{\rho} & =D u+T_{\alpha_{\rho}}^{\prime}\left(v_{\rho}-u\right)\left(D v_{\rho}-D u\right) \\
& =\left[1-T_{\alpha_{\rho}}^{\prime}\left(v_{\rho}-u\right)\right] D u+T_{\alpha_{\rho}}^{\prime}\left(v_{\rho}-u\right) D v_{\rho}
\end{aligned}
$$

and thus the convexity of $j(x, \cdot)$ yields

$$
\begin{aligned}
f_{\rho}\left(u_{\rho}\right) & =\int_{\Omega} j_{\rho}\left(x, D u_{\rho}(x)\right) d x \\
& \leqslant \int_{\Omega}\left[1-T_{\alpha_{\rho}}^{\prime}\left(v_{\rho}-u\right)\right] j_{\rho}(x, D u(x)) d x+\int_{\Omega} T_{\alpha_{\rho}}^{\prime}\left(v_{\rho}-u\right) j_{\rho}\left(x, D v_{\rho}(x)\right) d x
\end{aligned}
$$

Since $T_{\alpha}^{\prime}=1$ on $[-\alpha,+\alpha]$ and 0 outside, we get that

$$
f_{\rho}\left(u_{\rho}\right) \leqslant f_{\rho}\left(v_{\rho}\right)+\int_{\left\{\left|v_{\rho}-u\right| \geqslant \alpha_{\rho}\right\}} j_{\rho}(x, D u(x)) d x .
$$

Now recall that $u$ is in $H^{1}(\Omega)$ and that the integrands $j_{\rho}$ are uniformly majorized and thus

$$
f_{\rho}\left(u_{\rho}\right) \leqslant f_{\rho}\left(v_{\rho}\right)+\int_{\left\{\left|v_{\rho}-u\right|>\alpha_{\rho}\right\}} M\left(1+|D u|^{2}\right) d x .
$$

This with (2.12) yields

$$
f_{\rho}\left(u_{\rho}\right)-M \int_{\left\{\left|v_{\rho}-u\right|>\alpha_{\rho}\right\}}\left(1+|D u|^{2}\right) d x<\inf _{\|w-u\|_{\infty}<\eta} f_{\rho}(w)-\varepsilon .
$$

As $\rho \downarrow 0, u_{\rho}$ tends to $u$ in $\mathfrak{L}^{\infty}$. Thus for $\rho$ sufficiently small

$$
\inf _{\|w-u\|_{\infty}<\eta} f_{\rho}(w) \leqslant f_{\rho}\left(u_{\rho}\right)
$$

and hence for $\rho$ sufficiently small, from the two preceding inequalities we get

$$
0<\varepsilon<M \int_{\left\{\left|v_{\rho}-u\right|>\alpha_{\rho}\right\}}\left(1+|D u|^{2}\right) d x .
$$

Since $\left(1+|D(u)|^{2}\right) \in \mathcal{L}^{l}(\Omega)$ and meas $\left\{\left|v_{\rho}-u\right|>\alpha_{\rho}\right\} \downarrow 0$, the above yields a contradiction, which completes the proof.

The remainder of this section is devoted to the relationship between $\tau$-epi convergence and pointwise convergence. The pointwise-limit functions of a filtered collection of functions $\left\{f_{\nu}, \nu \in N\right\}$ are denoted by li $f_{\nu}$ and ls $f_{\nu}$ respectively and are defined by

$$
\text { li } f_{\nu}(x)=\sup _{H \in \mathcal{H}} \inf _{\nu \in H} f_{\nu}(x)
$$


and

$$
\text { ls } f_{\nu}(x)=\inf _{H \in \mathcal{K}} \sup _{\nu \in H} f_{\nu}(x)=\sup _{H \in \mathscr{H} C} \inf _{\nu \in H} f_{\nu}(x) .
$$

If $\iota$ denotes the discrete topology on $X$, then $\mathcal{G}_{\iota}(x)$ consists of all subsets of $X$ containing $x$. Whence

$$
\text { li } f_{\nu}=\mathrm{li}_{\iota} f_{\nu} \text { and } \mathrm{ls} f_{\nu}=1 \mathrm{~s}, f_{\nu} \text {, }
$$

from which it follows, via (1.14) and (1.15), that

$$
\operatorname{li}_{\tau} f_{\nu} \leqslant \operatorname{li} f_{\nu},
$$

and

$$
\text { ls } f_{\nu} \leqslant \operatorname{ls} f_{\nu} .
$$

Now, however, it is possible to simplify the definition of equi-lower semicontinuity.

Definition 2.17. A filtered collection of functions $\left\{f_{\nu}, \nu \in N\right\} \subset \bar{R}^{X}$ is $\tau$-equi-lower semicontinuous ( $\tau$-equi-1.sc.) at $x$ if there exists $\varepsilon_{x}>0$ such that

$\left(\mathrm{d}_{\mathrm{p}}\right)$ to every $\left.\varepsilon \in\right] 0, \varepsilon_{x}$ [ there correspond $H \in \mathcal{H}$ and $V \in \mathcal{G}_{\tau}(x)$ such that for all $\nu \in H$

$$
\inf _{y \in V} f_{\nu}(y) \geqslant \min \left[f_{\nu}(x)-\varepsilon, \varepsilon^{-1}\right] .
$$

The collection is said to be $\tau$-equi-l.sc. if $\left(\mathrm{d}_{\mathrm{p}}\right)$ is satisfied at every $x \in X$.

Clearly (d) implies $\left(\mathrm{d}_{\mathrm{p}}\right)$ since $\{x\} \in \mathcal{G}_{\iota}(x)$. On the other hand, given $\varepsilon>0$ and $W \in \mathcal{G}_{\imath}(x)$ (any set $W$ containing $x$ ), we always have that

$$
\inf _{y \in W} f_{\nu}(y)-\varepsilon \leqslant f_{\nu}(x)-\varepsilon .
$$

If $\left(\mathrm{d}_{\mathrm{p}}\right)$ is satisfied there exist $H \in \mathcal{H}$ and $V \in \mathcal{G}_{\tau}(x)$ such that

$$
f_{\nu}(x)-\varepsilon \leqslant \inf _{y \in V} f_{\nu}(y)
$$

for all $\nu \in H$. The two preceding inequalities yield (d).

In this setting Theorems 2.3 and 2.9 become

THEOREM 2.18. Suppose $\left\{f_{\nu}, \nu \in N\right\} \subset \bar{R}^{X}$ is a filtered collection of functions.

(i) If the collection is $\tau$-equi-l.sc., then $\operatorname{li}_{\tau} f_{\nu}=\operatorname{li} f_{\nu}$ and $\operatorname{ls}_{\tau} f_{\nu}=\operatorname{ls} f_{\nu}$.Also $f=\operatorname{lm} f_{\nu}$ if and only if $f=\operatorname{lm}_{\tau} f_{\nu}$.

(ii) If $-\infty<f=\operatorname{lm} f_{\nu}=\operatorname{lm}_{\tau} f_{\nu}$, then the collection is $\tau$-equi-l.sc.

By means of Proposition 2.2, and relying on the definition (1.16) of Mosco limit, we can obtain a whole slough of convergence results. For example,

Corollary 2.19. Convergence Theorem. Suppose that $\sigma \supset \tau$. If $f=\operatorname{lm} f_{\nu}$ and the $\left\{f_{\nu}, \nu \in N\right\}$ are $\tau$-equi-l.sc., then $f=\operatorname{lm}_{\tau, \sigma} f_{\nu}$. Also, if $f=\operatorname{lm}_{\sigma, \tau} f_{\nu}$ and the collection is $\tau$-equi-l.sc., then $f=\operatorname{lm} f_{v}$.

To verify $\tau$-equi-lower semicontinuity it is often convenient to decompose condition (d) into one that strictly requires equi-lower semicontinuity on some set $D \subset X$, and in another condition on the set $D$ itself that will imply uniform divergence on the complement of $D$. This approach had been followed in an earlier version of this 
article. (Note that the same ideas can be used in the more general context of $\tau / \sigma$-equi-lower semicontinuity.)

THEOREM 2.20. Suppose $\left\{f_{\nu}, \nu \in N\right\} \subset \bar{R}^{X}$ is a filtered collection of $\tau$-lower semicontinuous functions. It is $\tau$-equi-lower semicontinuous if and only if there exists a set $D$ with dom $\operatorname{li}_{\tau} f_{\nu} \subset D \subset X$ such that

$\left(\mathrm{d}_{\mathrm{p}}^{\prime}\right)$ to every pair $x \in D, \varepsilon>0$, there corresponds $H \in \mathcal{H}, V \in \mathcal{G}_{\tau}(x)$ such that for all $\nu \in H, \inf _{y \in V} f_{\nu}(y) \geqslant f_{\nu}(x)-\varepsilon$.

Proof. Since $\left(\mathrm{d}_{\mathrm{p}}\right)$ implies $\left(\mathrm{d}_{\mathrm{p}}^{\prime}\right)$ on any set $D$ which includes dom $\mathrm{li}_{\tau} f_{\nu}$, the "only if" part is trivially satisfied. To obtain the converse simply note that if $x \notin \operatorname{dom~li}_{\tau} f_{\nu}$, it means that given any $\varepsilon>0$, there exist $H_{\varepsilon} \in \mathcal{H}$ and $V_{\varepsilon} \in \mathcal{G}_{\tau}(x)$ such that for all $\nu \in H_{\varepsilon}$,

$$
\inf _{y \in V_{\mathrm{f}}} f_{\nu}(y) \geqslant \varepsilon^{-1} .
$$

Now recall that $\operatorname{li}_{\tau} f_{\nu}(x) \leqslant \operatorname{li} f_{\nu}(x)$ which implies that there exists $H^{\prime} \in \mathcal{H}$ such that $f_{\nu}(x)-\varepsilon \geqslant \varepsilon^{-1}$ for all $\nu \in H^{\prime}$. Hence we have that for all $\nu \in H_{\varepsilon} \cap H^{\prime}(\in \mathcal{H})$,

$$
\inf _{y \in V_{\varepsilon}} f_{\nu}(y) \geqslant \min \left[f_{\nu}(x)-\varepsilon, \varepsilon^{-1}\right] \text {. }
$$

Thus $\left(\mathrm{d}_{\mathrm{p}}\right)$ holds for every $x \notin \operatorname{dom~li}_{\tau} f_{\nu}$.

Since dom $\operatorname{li}_{\tau} f_{\nu} \subset D$, we know that $\left(\mathrm{d}_{\mathrm{p}}^{\prime}\right)$ is satisfied for every $x \in \operatorname{dom~li}_{\tau} f_{\nu}$, i.e. for all $\varepsilon>0$ there exist a pair $H_{\varepsilon} \in \mathcal{H}$ and $V_{\varepsilon} \in \mathcal{G}_{\tau}(x)$ such that for all $\nu \in H$,

$$
\inf _{y \in V_{\varepsilon}} f_{\nu}(y) \geqslant f_{\nu}(x)-\varepsilon \text {. }
$$

On the other hand, $x \in \operatorname{dom~li}_{\tau} f_{\nu}$ implies that $\operatorname{li}_{\tau} f_{\nu}(x)<\infty$. Pick $\varepsilon_{x}>0$ such that $\varepsilon_{x}^{-1}>\operatorname{li}_{\tau} f_{\nu}(x)$. Then by the definition (1.1) of $\operatorname{li}_{\tau} f_{\nu}$ it follows that for every $\left.\varepsilon \in\right] 0, \varepsilon_{x}[$, every $H \in \mathcal{H}$ and $V \in \mathcal{G}_{\tau}(x)$,

$$
\inf _{\nu \in H} \inf _{y \in V} f_{\nu}(y)<\varepsilon^{-1} .
$$

This and the $\tau$-lower semicontinuity of the $f_{\nu}$ at $x$ implies that for all $\left.\varepsilon \in\right] 0, \varepsilon_{x}[$,

$$
f_{\nu}(x)-\varepsilon \leqslant \varepsilon^{-1} \text {. }
$$

Thus also when $x \in \operatorname{dom~li}_{\tau} f_{\nu}$ with the $H_{\varepsilon}$ and $V_{\varepsilon}$ identified as above, we have that for all $\nu \in H_{\varepsilon}$,

$$
\inf _{y \in V_{\varepsilon}} f_{\nu}(y) \geqslant \min \left[f_{\nu}(x)-\varepsilon, \varepsilon^{-1}\right]
$$

for all $\varepsilon \in] 0, \varepsilon_{x}$ [ for some $\varepsilon_{x}>0$, i.e. $\left(\mathrm{d}_{\mathrm{p}}\right)$ also holds on dom li $f$.

The assertions of Theorem 2.18 remain valid with a weakened version of $\tau$ equi-lower semicontinuity, when $X$ is linear, $\tau$ is locally convex and the $\left\{f_{\nu}, \nu \in N\right\}$ are convex functions. This is the content of Theorem 2.24. To start off with we need to establish the convexity of some limit functions which we obtain as a corollary of the next lemma.

Lemma 2.21. Suppose $\left\{C_{\nu}, \nu \in N\right\}$ is a filtered collection of convex subsets of a locally convex linear space. Then $\mathrm{Li} \mathrm{C}_{\nu}$ is convex. 
Proof. Clearly, $L i C_{\nu}$ is trivially convex if $L i C_{\nu}=\varnothing$. So let us assume that $\mathrm{Li} C_{\nu} \neq \varnothing$. From the definition (1.6) of $\mathrm{Li} C_{\nu}$, it follows that $x \in \mathrm{Li} C_{\nu}$ if and only if to every neighborhoods $V$ of $x$, there corresponds $H \in \mathcal{H}$ such that for all $\nu \in H$

$$
C_{\nu} \cap V \neq \varnothing \text {. }
$$

Take $x^{0}, x^{1} \in L i C_{\nu}$ and for $\lambda \in[0,1]$ define $x^{\lambda}=(1-\lambda) x^{0}+\lambda x^{1}$. We need to show that if $V^{\lambda}$ is a neighborhood of $x^{\lambda}$, there exists $H^{\lambda} \in \mathcal{H}$ such that $C_{\nu} \cap V^{\lambda} \neq \varnothing$ for all $\nu \in H^{\lambda}$. In view of local convexity we only need to show this for convex neighborhoods of $x^{\lambda}$ and henceforth we take it for granted that $V^{\lambda}$ is convex. Define

$$
V^{0}=V^{\lambda}+x^{0}-x^{\lambda} \text { and } V^{1}=V^{\lambda}+x^{1}-x^{\lambda} \text {. }
$$

These are (convex) neighborhoods of $x^{0}$ and $x^{1}$ and thus there exist $H^{0}$ and $H^{1}$ such that (2.22) is satisfied. Let $H^{0} \cap H^{1}=H(\in \mathcal{H})$ and since for all $\nu \in H^{\lambda}$,

$$
V^{0} \cap C_{\nu} \neq \varnothing \quad \text { and } \quad V^{1} \cap C_{\nu} \neq \varnothing,
$$

it follows from the construction of $V^{0}$ and $V^{1}$, and the convexity of the $C_{\nu}$ and of $V^{\lambda}$ that $V^{\lambda} \cap C_{\nu} \neq \varnothing$ for all $\nu \in H^{\lambda}$.

Corollary 2.23. Suppose $\left\{f_{\nu}, \nu \in N\right\}$ is a filtered collection of convex functions defined on the locally convex linear space $(X, \tau)$. Then $\mathrm{ls}_{\tau} f_{\nu}$ is a convex function, and if they exist so are $\operatorname{lm}_{\tau} f_{\nu}$ and $\operatorname{lm} f_{\nu}$.

Proof. Recall that a function is convex if and only if its epigraph is convex. Thus the convexity of $\mathrm{ls}_{\tau} f_{\nu}$ follows from (1.11) and Proposition 2.21 since by assumption all the $\left\{\right.$ epi $\left.f_{\nu}, \nu \in N\right\}$ are convex. If $\operatorname{lm}_{\tau} f_{\nu}$ exists, then $\operatorname{lm}_{\tau}=1 \mathrm{~s}_{\nu}$, and the convexity of $\operatorname{lm}_{\tau}$ follows from the above. The convexity of $\operatorname{lm} f_{\nu}$ can be verified directly or follows from the above since, if it exists, $\operatorname{lm} f_{v}=\operatorname{lm}_{\imath} f_{v}=1 \mathrm{~s}_{\imath} f_{\nu}$.

Note, however, that in general $\operatorname{li}_{\tau} f_{\nu}$ is not convex, although the $f_{\nu}$ are convex. Consider, for example $\left\{f_{\nu}, \nu=1,2, \ldots\right\}$ such that for $k=1,2, \ldots$,

$$
f_{2 k}(x)=|x-1| \text { and } f_{2 k-1}(x)=|x+1| \text {. }
$$

Then clearly $\mathrm{li}_{\tau} f_{\nu}$ is not convex, since

$$
\operatorname{li}_{\tau} f_{\nu}= \begin{cases}|x+1| & \text { if } x \leqslant 0, \\ |x-1| & \text { if } x \geqslant 0 .\end{cases}
$$

THEOREM 2.24. Suppose $\left\{f_{\nu}, \nu \in N\right\} \subset \bar{R}^{X}$ is a filtered collection of $\tau$-lower semicontinuous convex functions defined on a locally convex linear space $X$. Moreover, suppose that either $-\infty<\operatorname{lm}_{\tau} f_{\nu}$ exists or $-\infty<\operatorname{lm} f_{\nu}$ exists and is $\tau$-l.sc. Then the collection $\left\{f_{\nu}, \nu \in N\right\}$ is $\tau$-equi-l.sc. if and only if there exists $D \subset X$ such that conditions $\left(\mathrm{d}_{\mathrm{p}}^{\prime}\right)$ and $\left(\sim \mathrm{d}_{\mathrm{c}}^{\prime}\right)$ are satisfied, where

$\left(\sim \mathrm{d}_{\mathrm{c}}^{\prime}\right)$ to every pair $x \notin \mathrm{cl} D, a \in R$ there corresponds $H \in \mathcal{H}, V \in \mathcal{G}_{\tau}(x)$ such that for all $\nu \in H$,

$$
\inf _{y \in V} f_{\nu}(y) \geqslant a .
$$

Proof. Since $\iota \supset \tau$, from Theorem 2.9 it follows that we may as well prove that the conditions imply that $\operatorname{lm} f_{\nu}=\operatorname{lm}_{\tau} f_{\nu}$. The converse being trivially true, since 
$\tau$-equi-lower semicontinuity implies, via Theorem 2.20, that dom $\operatorname{li}_{\tau} f_{\nu} \subset D$ and that $\left(\mathrm{d}_{\mathrm{p}}^{\prime}\right)$ holds, and dom $\mathrm{li}_{\tau} f_{\nu} \subset D$ yields $\left(\sim \mathrm{d}_{\mathrm{c}}^{\prime}\right)$, in fact not just for every pair $x \notin \mathrm{cl} D$, $a \in R$ but for every pair $x \notin D, a \in R$.

From the proof of Theorem 2.3, with $\sigma=\imath$, we see that with $D=\operatorname{dom} \operatorname{li}_{\tau} f_{\nu},\left(\mathrm{d}_{\mathrm{p}}^{\prime}\right)$ implies that $\operatorname{li} f_{\nu} \leqslant \operatorname{li}_{\tau} f_{\nu}$ and $\mathrm{ls}_{\nu} \leqslant \mathrm{ls}_{\tau} f_{\nu}$ on $D$. Similarly $\left(\sim \mathrm{d}_{\mathrm{c}}^{\prime}\right)$ yields the same relations on $X \backslash \mathrm{cl} D$. Combining this with (2.15) and (2.16), we have that $\left(\mathrm{d}_{\mathrm{p}}^{\prime}\right)$ and $\left(\sim \mathrm{d}_{\mathrm{c}}^{\prime}\right)$ yield on $X \backslash Q$, with $Q=\operatorname{cl} D \backslash D$,

$$
\operatorname{li}_{\tau} f_{\nu}=\operatorname{li} f_{\nu} \text { and } \quad \mathrm{ls}_{\tau} f_{\nu}=\mathrm{ls} f_{\nu} .
$$

Moreover, $\mathrm{ls}_{\tau} f_{\nu}$ is convex, and so are $\operatorname{lm}_{\tau} f_{\nu}$ and $\operatorname{lm} f_{\nu}$ if they exist, cf. Corollary 2.23.

If $-\infty<f:=\operatorname{lm} f_{\nu}$ exists and is $\tau$-l.sc., it follows from the above that $f=1 \mathrm{~s}_{\tau} f_{\nu}=$ $\operatorname{li}_{\tau} f_{\nu}$ on $X \backslash Q$. Convexity also yields equality on $Q$ as we now demonstrate. Suppose that $x^{1} \in Q$ and take $x^{0} \in \operatorname{dom} f \subset D$, and without loss of generality assume that $f\left(x^{0}\right)=0$. Given any $\varepsilon>0, G \in \mathcal{S}_{\tau}\left(x^{1}\right)$, and $H \in \mathcal{K}$, the definition of $\mathrm{li}_{\tau}$ yields $\nu_{\varepsilon} \in H$ and $y_{\varepsilon} \in G$ such that

$$
\alpha=\operatorname{li}_{\tau} f_{\nu}\left(x^{1}\right) \geqslant f_{\nu}\left(y_{\varepsilon}\right)-\varepsilon .
$$

For $\lambda \in[0,1]$, define

$$
x_{H, G}^{\lambda}=(1-\lambda) x^{0}+\lambda y_{\varepsilon} .
$$

The convexity of the $f_{\nu}$ implies that

$$
f_{\nu_{\varepsilon}}\left(x_{H, G}^{\lambda}\right) \leqslant(1-\lambda) f_{\nu_{\varepsilon}}\left(x^{0}\right)+\lambda f_{\nu_{\varepsilon}}\left(y_{\varepsilon}\right) \leqslant(1-\lambda) f_{\nu_{\varepsilon}}\left(x^{0}\right)+\lambda(\alpha+\varepsilon) .
$$

Now note that for any fixed $\lambda \in[0,1], x^{\lambda}=(1-\lambda) x^{0}+\lambda x^{1}$ is a limit point of the filtered collection $\left\{x_{H, G}^{\lambda},(H, G) \in\right.$ filter generated by $\left.\mathcal{H} \times \mathcal{G}_{\tau}\left(x^{1}\right)\right\}$. Hence for every $\lambda \in[0,1[$

$$
f\left(x^{\lambda}\right) \leqslant \operatorname{li}_{\tau} f_{\nu}\left(x^{\lambda}\right) \leqslant(1-\lambda) f\left(x^{0}\right)+\lambda \alpha=\lambda \alpha .
$$

Let $\lambda \uparrow 1$. From the lower semicontinuity of $f$, we get that $f\left(x^{1}\right) \leqslant \mathrm{li}_{\tau} f_{\nu}\left(x^{1}\right)$. From which it follows that $f \leqslant \operatorname{li}_{\tau} f_{\nu} \leqslant \operatorname{ls}_{\tau} f_{\nu}$ on $Q$ which implies that $f=\operatorname{lm}_{\tau} f_{\nu}$ on $X$ since (2.16) holds universally.

Now consider the case when $-\infty<f:=\operatorname{lm}_{\tau} f_{\nu}$ exists. From the observations at the beginning of the proof, in particular (2.25), it follows that

$$
f=\operatorname{li} f_{\nu}=\operatorname{ls} f_{\nu} \text { on } X \backslash Q \text {. }
$$

It suffices to show that $f>\operatorname{ls} f_{\nu}$ on $Q$ since (2.15) and (1.5) imply that $f \leqslant \operatorname{li} f_{\nu} \leqslant \operatorname{ls} f_{\nu}$. Again let $x^{1} \in Q$ and $x^{0} \in \operatorname{dom} f \subset D$, and without loss of generality assume that $f\left(x^{0}\right)=0$. Given any $\varepsilon>0$ and $H \in \ddot{\mathcal{H}}$, the definition (2.14) of ls yields $\nu_{\varepsilon} \in H$ such that

$$
\text { ls } f_{\nu}\left(x^{1}\right) \geqslant f_{\nu}\left(x^{1}\right)-\varepsilon .
$$

Set $x^{\lambda}=(1-\lambda) x^{0}+\lambda x^{1}$ for $\lambda \in[0,1]$. Now the convexity of the $f_{\nu}$ implies that for all $\lambda \in[0,1[$,

$$
\begin{aligned}
f_{\nu_{\varepsilon}}\left(x^{\lambda}\right) & \leqslant(1-\lambda) f_{\nu_{\varepsilon}}\left(x^{0}\right)+\lambda f_{\nu_{\varepsilon}}\left(x^{1}\right) \\
& \leqslant(1-\lambda) f_{\nu_{\varepsilon}}\left(x^{0}\right)+\lambda \operatorname{ls} f_{\nu}\left(x^{1}\right)+\lambda \varepsilon .
\end{aligned}
$$


Taking limits (with respect to $\ddot{\mathscr{C}}$ ) we get that

$$
f\left(x^{\lambda}\right) \leqslant(1-\lambda) f\left(x^{0}\right)+\lambda \text { ls } f_{\nu}\left(x^{1}\right)+\lambda \varepsilon .
$$

Let $\lambda \uparrow 1$. From the lower semicontinuity of $f=\operatorname{lm}_{\tau} f_{\nu}$ (Corollary 1.12) and the fact that $\varepsilon$ is arbitrary it follows that $f\left(x^{1}\right) \leqslant 1$ s $f_{\nu}\left(x^{1}\right)$, which completes the proof.

This last theorem allows us to relate the definition of $\tau$-equi-l.sc. used here and the original definition suggested by Salinetti and Wets [1977] for sequences of $\tau$-1.sc. (proper) convex functions defined on a reflexive Banach space. The sequence $\left\{f_{\nu}\right.$, $\nu=1, \ldots\}$ was said to be $\tau$-equi-l.sc. with respect to a proper $\tau$-l.sc. convex function $f$ if

( $\alpha$ ) for all $x \in \operatorname{dom} f$ and $\varepsilon>0$, there exist $V \in \mathcal{G}_{\tau}(x)$ and $\nu_{\varepsilon}$ such that for all $\nu \geqslant \nu_{\varepsilon}$

$$
\inf _{y \in V} f_{\nu}(y) \geqslant f_{\nu}(x)-\varepsilon
$$

( $\beta$ ) for all $x \in \operatorname{dom} f$, there exists $\nu_{x}$ such that for all $\nu \geqslant \nu_{x}, x \in \operatorname{dom} f_{\nu}$;

$(\gamma)$ the $f_{\nu}$ converge uniformly to $+\infty$ on every compact subset of $X \backslash$ cl dom $f$.

If we choose $f$ and $D$ such that $D=\operatorname{dom} f$ then $\left(\mathrm{d}_{\mathrm{p}}^{\prime}\right)$ is precisely $(\alpha)$. And clearly $\left(\sim \mathrm{d}_{\mathrm{c}}^{\prime}\right)$ implies $(\gamma)$, but also the converse holds because the closed balls of a reflexive Banach space are weakly compact. Condition $(\beta)$ is superfluous because it is automatically satisfied if the functions $f_{\nu}$ have $f$ as a pointwise limit (Salinetti and Wets [1977, Lemma 2.ii]) and it is implied by $(\alpha)$ if the sequence $f_{v}$ epi-converges. Thus Theorems 1, 2 and 3 of Salinetti and Wets [1977] are special cases of Theorem 2.24.

Bernard-Mazure [1981] also suggests a definition of $\tau$-equi-l.sc. In our framework, her definition reads: A filtered collection $\left\{f_{\nu}, \nu \in N\right\}$ of extended real-valued functions is $\tau$-equi-1.sc. if for every $x \in \operatorname{dom~li}_{\sigma} f_{\nu}$ and every $\varepsilon>0$ there exists $H \in \mathcal{H}$ and $V \in \mathcal{G}_{\boldsymbol{\sigma}}(x)$ such that for all $\nu \in H$,

$$
\inf _{y \in V} f_{\nu}(y) \geqslant f_{\nu}(x)-\varepsilon .
$$

The difference with Definition 2.17 is that here the effective domain of $\mathrm{li}_{\sigma} f$ must be calculated, a not necessarily easy task. Finally we note that Buttazzo [1977] in his Proposition (2.1) introduces a sufficient condition to obtain the identities

$$
\operatorname{li}_{\sigma} f_{\nu}=\operatorname{li} f_{\nu} \text { and } \quad \mathrm{ls}_{\sigma} f_{\nu}=\mathrm{ls} f_{\nu} \text {. }
$$

In order to verify his condition, the functions $f_{\nu}$ must be finite valued. His result however can be viewed as a forerunner of Corollary 2.19.

III. The hyperspace of closed sets. Let $(Y, \eta)$ be a topological space. In this section we have collected some facts about the (hyper) space of closed subsets of $Y$ equipped with the topology of set-convergence, as defined by (1.8). This turns out to be a variant of the Vietoris finite topology, at least when $(Y, \eta)$ is separated (Hausdorff) and locally compact. Most of the results found below can be extracted from articles by Choquet [1947-1948], and by Michael [1951] and from the book by Kuratowski [1950]. 
By $\mathscr{F}_{Y}$, or simply $\mathscr{F}$ if no confusion is possible, we denote the hyperspace of closed subsets of $Y$. The topology $\mathcal{T}$ of $\mathcal{F}$ is generated by the subbase of open sets:

$$
\left\{\mathscr{F}^{K}, K \in \mathscr{K}_{\mathcal{K}}\right\} \quad \text { and } \quad\left\{\mathscr{F}_{G}, G \in \mathcal{G}\right\}
$$

where $\mathscr{K}$ and $\mathcal{G}$ are the hyperspaces of compact and open subsets of $Y$ respectively, and for any $Q \subset Y$,

$$
\mathscr{F}^{Q}=\{F \in \mathscr{F} \mid F \cap Q=\varnothing\} \quad \text { and } \quad \mathscr{F}_{Q}=\{F \in \mathscr{F} \mid F \cap Q \neq \varnothing\} .
$$

Proposition 3.1. Suppose that $Y$ is separated and locally compact, $\left\{C_{\nu}, \nu \in N\right\}$ is a filtered collection of subsets of $Y$, and $C \subset Y$ is closed. Then

(i) $C \subset \mathrm{Li} C_{\nu}$ if and only if to every $G \in \mathcal{G}$ such that $C \cap G \neq \varnothing$, there corresponds $H_{G} \in \mathcal{H}$ such that for every $\nu \in H_{G}, C_{\nu} \cap G \neq \varnothing$;

(ii) $C \supset L s C_{\nu}$ if and only if to every $K \in \mathcal{K}$ such that $C \cap K=\varnothing$, there corresponds $H_{K} \in \mathcal{H}$ such that for every $\nu \in H_{K}, C_{\nu} \cap K=\varnothing$.

Moreover, $C=\operatorname{Lm} C_{\nu}$ if and only if $C=\mathcal{T}-\lim C_{\nu}$.

Proof. It will be sufficient to prove (i) and (ii) since the last assertion follows immediately from (i) and (ii) and the construction of $\mathcal{T}$.

Suppose first that $x \in C$, then $C \cap G \neq \varnothing$ for all $G \in \mathcal{G}_{\eta}(x)$. The "if" part of (i), implies that $C_{\nu} \cap G \neq \varnothing$ for all $\nu \in H_{G}$ with $H_{G} \in \mathcal{H}$. Every $H^{\prime}$ in $\mathcal{H}$ meets every $H \in \ddot{\mathcal{H}}$ and hence

$$
\left(\bigcup_{\nu \in H} C_{\nu}\right) \cap G \neq \varnothing
$$

for every $H \in \ddot{\mathscr{H} C}$ and $G \in \mathcal{G}_{\eta}(x)$. Thus for every $H \in \ddot{\mathscr{H}}, x \in \operatorname{cl}\left(\cup_{\nu \in H} C_{\nu}\right)$ and consequently by (1.6) $x \in \mathrm{Li} C_{\nu}$, i.e. $C \subset \mathrm{Li} C_{\nu}$.

If $C \subset \operatorname{Li} C_{\nu}$, then $C \cap G \neq \varnothing$ implies that $G \cap\left(\cap_{H \in \ddot{\mathcal{K}}} \operatorname{cl}\left(\cup_{\nu \in H} C_{\nu}\right)\right) \neq \varnothing$, i.e., for every $H \in \ddot{\mathscr{K}}$,

$$
\left(\bigcup_{\nu \in H} C_{\nu}\right) \cap G \neq \varnothing
$$

or equivalently there exists $H_{G} \in \mathcal{H}$ such that for all $\nu \in H_{G}, C_{\nu} \cap G \neq \varnothing$, again because $\mathscr{H}$ consists of all the subsets of $N$ that meet every set in $\ddot{\mathscr{H}}$. This completes the proof of (i).

Suppose that $x \in L s C_{\nu}$, then for every $H \in \mathcal{H}, x \in \operatorname{cl}\left(\cup_{\nu \in H} C_{\nu}\right)$, cf. (1.7). If $x \notin C$, by local compactness of $Y$, there is a compact neighborhood $K$ of $x$ such that $K \cap C=\varnothing$. The "if" part of (ii) then implies that $K \cap\left(\cup_{\nu \in H_{K}} C_{\nu}\right)=\varnothing$ for some $H_{K} \in \mathcal{H}$, i.e., $x \notin \operatorname{cl}\left(\bigcup_{\nu \in H_{K}} C_{\nu}\right)$ contradicting the assumption that $x \in L s C_{\nu}$.

Now suppose that $C \supset L s C_{\nu}, C \cap K=\varnothing$, but for every $H \in \mathcal{H}$ we can find $\nu \in H$ such that $C_{\nu} \cap K \neq \varnothing$, i.e. there exists $H^{\prime} \in \ddot{\mathscr{H} C}$ such that $C_{\nu} \cap K \neq \varnothing$ for every $\nu \in H^{\prime}$. Since $K$ is compact, it follows that the $\left\{C_{\nu} \cap K, \nu \in H^{\prime}\right\}$ admit at least one cluster point $x \in K$. Then for every $H \in \mathcal{K}$,

$$
x \in \operatorname{cl}\left(\bigcup_{\nu \in H} C_{\nu}\right) \cap K,
$$


and consequently, $x \in L s C_{\nu} \cap K$. But this contradicts the assumption that $C \supset$ Ls $C_{\nu}$.

Thus $\mathcal{T}$ is indeed the topology of set-convergence as defined in $\S I$. The next proposition yields the properties of $(\mathcal{F}, \mathcal{T})$ that are needed in the sequel.

Proposition 3.2. Suppose that $Y$ is separated (Hausdorff) and locally compact. Then $(\mathscr{F}, \mathcal{T})$ is regular and compact.

Proof. By construction the sets $\left\{\mathscr{F}_{K} ; K \in \mathscr{K}\right\}$ and $\left\{\mathscr{F}^{G} ; G \in \mathcal{G}\right\}$ are the complements of open (base) sets, and thus are closed. In particular, this implies that singletons are closed, since

$$
F=\left(\bigcap_{y \in F} \mathscr{F}_{\{y\}}\right) \cap \mathscr{F}^{G},
$$

$G=Y \backslash F$ is open.

To see that $(\mathscr{F}, \mathcal{T})$ is separated, let $F_{1}$ and $F_{2}$ be two subsets of $\mathscr{F}$ such that $F_{1} \neq F_{2}$. Then there is some $y$ that belongs to $F_{1}$ but not to $F_{2}$ (or vice-versa). Since $Y$ is locally compact by assumption and $F_{2}$ is closed, there exists $K^{0}$, an open precompact neighborhood of $y$, such that $K=\mathrm{cl} K^{0}$ is disjoint of $F_{2}$. Hence

$$
F_{1} \in \mathscr{F}_{K^{0}} \quad \text { and } \quad F_{2} \in \mathscr{F}^{K} .
$$

The compactness of $(\mathscr{F}, \widetilde{T})$ follows from Alexander's characterization of compactness in terms of the finite intersection property of a subbase of closed (hyper)sets. Suppose that

$$
\left(\bigcap_{i \in I} \mathcal{F}_{K_{i}}\right) \cap\left(\bigcap_{j \in J} \mathcal{F}_{\mathcal{F}} G_{j}\right)=\varnothing
$$

where $K_{i} \in \mathfrak{T}, G_{j} \in \mathcal{G}$ and $I$ and $J$ are arbitrary index sets. We must show that the family of sets $\left\{K_{i}, i \in I ; G_{j}, j \in J\right\}$ contains a finite subfamily that has an empty intersection. Let $G=\cup_{j \in J} G_{j}$ and note that $G \in \mathcal{G}$. Now observe that (3.3) holds if and only if

$$
\bigcap_{i \in I}\left(\mathscr{F}_{K} \cap \widetilde{\mathscr{F}}^{G}\right)=\varnothing
$$

or still, if and only if for some $i_{0} \in I, \mathscr{F}_{K_{t_{0}}} \cap \mathscr{F}^{G}=\varnothing$, or equivalently, if and only if there exists $i_{0} \in I$ such that

$$
K_{i_{0}} \subset G \text {. }
$$

But $K_{i_{0}}$ is compact and thus the open cover $\left\{G_{j}, j \in J\right\}$ contains a finite subcover $\left\{G_{j_{1}}, \ldots, G_{j_{q}}\right\}$. Hence (3.3) holds if and only if

$$
\mathscr{F}_{K_{\iota_{0}}} \cap\left(\bigcap_{i=1}^{q} \mathscr{F}^{G_{I_{i}}}\right)=\varnothing .
$$

Since $(\widetilde{F}, \widetilde{T})$ is compact and separated, it is also regular.

IV. Compactness criteria for spaces of semicontinuous functions. The relationship between pointwise- and $e_{\tau}$-limits through equi-semicontinuity suggests a number of compactness criteria for spaces of semicontinuous and continuous functions, the 
celebrated Arzela-Ascoli Theorem being a special case of these. Our approach in fact provides an unconventional proof of this classical result.

Although a few of the (weaker) subsequent statements remain valid in a more general setting, we shall assume henceforth that the domain-space $(X, \tau)$ is separated and locally compact. Let $S C(X)$ be the space of $\tau$-l.sc. functions with range $\bar{R}$ and domain $X$. The elements of $S C(X)$ are in one-to-one correspondence with the elements of $\mathcal{E}$, the hyperspace of epigraphs, i.e. the closed subsets $E$ of $Y=X \times R$ such that $(x, a) \in E$ implies that $(x, b) \in E$ for all $b \geqslant a$. Note that $\{\varnothing\} \in \mathcal{E}$ and corresponds to the (continuous) function $f \equiv+\infty$. $\delta$ is a subset of $\mathscr{F}_{Y}$, the hyperspace of closed subsets of $Y=X \times R$.

Proposition 4.1. Suppose that $(X, \tau)$ is separated and locally compact. Then $\mathcal{E} \subset \mathscr{F}_{Y}$ is compact with respect to the $\mathcal{T}$ topology. Moreover, the $\mathcal{T}_{-}$-relative topology on $\mathcal{E}$ can be generated by the subbase of open sets:

$$
\left\{\mathcal{E}^{K, a} ; K \in \mathscr{K}_{X}, a \in R\right\} \quad \text { and } \quad\left\{\mathcal{E}_{G, a^{0}} ; G \in \mathcal{G}_{X}, a \in R\right\} \text {, }
$$

where for any $Q \subset X$ and $a \in \bar{R}$,

$$
\left.\left.\mathcal{E}^{Q, a}=\{E \in \mathcal{E} \mid E \cap(Q x]-\infty, a]\right)=\varnothing\right\}
$$

and

$$
\mathcal{E}_{Q, a^{0}}=\{E \in \mathcal{E} \mid E \cap(Q x]-\infty, a[) \neq \varnothing\} .
$$

Proof. Suppose $F \in \mathscr{F}_{Y} \backslash \mathcal{E}$, then there exist $x \in X$ and $a<b$ such that $(x, a) \in F$ but $(x, b) \notin F$. The local compactness of $X$ yields an open precompact set $K^{0}$ such that

$$
\mathscr{F}^{K \times\{b\}} \cap \mathscr{F}_{\left.K^{0} \times\right] a-\varepsilon, a+\varepsilon},
$$

with $K=\operatorname{cl} K^{0}$ and $0<\varepsilon<b-a$, is an open neighborhood of $F$ that does not contain any epigraphs. Thus $\mathscr{F} \backslash \mathcal{E}$ is open or equivalently $\mathcal{E}$ is closed. Since $\mathscr{F}$ is compact, so is $\varepsilon$.

To see that the $\mathcal{T}$-relative topology on $\mathcal{E}$ can be generated by the subbase described above, note that the topological properties of $Y=X \times R$ imply that the sets of the type

$$
\left\{\mathscr{F}^{K \times[a, b]} ; K \in \mathscr{K}_{X}, a, b \in R\right\} \quad \text { and } \quad\left\{\mathscr{F}_{G \times] a, b[} ; G \in \mathcal{G}_{X}, a, b \in R\right\}
$$

also are a subbase for $\mathcal{T}$ on $\mathscr{F}_{Y}$. The restriction of this subbase to $\mathcal{E}$, yields

$$
\varepsilon^{K \times[a, b]}=\varepsilon^{K, a} \quad \text { and } \quad \varepsilon_{G \times] a, b]}=\varepsilon_{G, a^{0}} .
$$

Combining Propositions 3.2 and 4.1 we get

COROLlaRY 4.2. The topological space $(\mathcal{E}, \mathcal{J})$ is regular and compact.

From Propositions 1.9, 3.1 and 4.1, with $e_{\tau}$ the topology of epi-convergence on $S C(X)$, we also get

COROLlaRy 4.3. The topological space $\left(S C(X), e_{\tau}\right)$ is regular and compact. 
The above implies that any closed subset of $S C$ is compact. In particular, note that for any $a \in R$ and $D \subset X$, the set

$$
S C^{a}(D)=\{f \in S C \mid f \leqslant a \text { on } D\}=\bigcap_{x \in D}\{f \in S C \mid f(x) \leqslant a\}
$$

is compact. To see this simply observe that $\{f \in S C \mid f(x) \leqslant a\}$ is closed since it corresponds in $\varepsilon$ to the $\mathcal{T}$-closed set

$$
\{E \in \varepsilon \mid E \cap(\{x\} \times]-\infty, a]) \neq \varnothing\} .
$$

Also, for any $a \in R$ and any open $G \in X$, the set

$$
S C_{a}(G)=\{f \in S C \mid f \geqslant a \text { on } G\}
$$

is closed since it corresponds in $\mathcal{E}$ to the $\widetilde{\mathcal{T}}$-closed set

$$
\{E \in \varepsilon \mid E \cap(G \times]-\infty, a[)=\varnothing\} .
$$

We have just shown that

COROllary 4.4. Any bounded collection of $\tau$-l.sc. functions is a compact subset of $\left(S C(X), e_{\tau}\right)$.

The topological space $(S C, p)$ is the space of $\tau$-l.sc. functions equipped with the topology of pointwise convergence. We already know that neither pointwise nor epigraph-convergence implies the other. However, in view of Theorem 2.15, these topologies coincide on $\tau$-equi-l.sc. subsets of $S C$.

Definition 4.5. A set $Q \subset S C(X)$ is $\tau$-equi-lower semicontinuous, or simply equi-1.sc. if to every $x \in X$ and $\varepsilon>0$ sufficiently small there correspond $V \in \mathcal{G}_{\tau}(x)$ such that for all $f$ in $\Theta$,

$$
\inf _{y \in V} f(y) \geqslant \min \left[f(x)-\varepsilon, \varepsilon^{-1}\right] .
$$

THEOREM 4.6. Suppose that $(X, \tau)$ is separated and locally compact. Then any $\tau$-equi-l.sc. family of $\tau$-l.sc. functions contains a (filtered) subfamily converging pointwise to a $\tau$-l.sc. function. Moreover, if the family of functions is bounded, it contains a subfamily converging pointwise to a bounded $\tau$-l.sc. function.

Proof. As follows from Theorem 2.18, for $\tau$-equi-l.sc. subsets of $S C(X)$, the $p$-closure or $e_{\tau}$-closure coincide. The first statement then follows from Corollary 4.3 and the second from Corollary 4.4 .

Every property derived for $\left(S C(X), e_{\tau}\right)$ has its counterpart in $\left(-S C(X),-e_{\tau}\right)$, the space of $\tau$-upper semicontinuous functions ( $\tau$-u.sc.) with the topology $-e_{\tau}$ of hypo (graph)-convergence. In particular, $\left(-S C(X),-e_{\tau}\right)$ is compact and any bounded subfamily is precompact. And thus, any $\tau$-equi-u.sc. family of (bounded) u.sc. functions contains a subfamily converging pointwise to a (bounded) $\tau$-u.sc. function.

Given $\left\{f_{\nu}, \nu \in N\right\}$ a filtered collection of functions, the $-e_{\tau}$-limit inferior is $-\left(\mathrm{ls}_{\tau}-f_{\nu}\right)$ and the $-e_{\tau}$-limit superior is $-\left(\mathrm{li}_{\tau}-f_{\nu}\right)$. The hypographs of these functions being precisely $L i$ hypo $f_{\nu}$ and $L s$ hypo $f_{\nu}$. We always have that

$$
\operatorname{li}_{\tau} f_{\nu} \leqslant \operatorname{li} f_{\nu}=-\left(\mathrm{ls}-f_{\nu}\right) \leqslant-\left(\mathrm{ls}_{\tau}-f_{\nu}\right)
$$


and

$$
\mathrm{ls}_{\tau} f_{\nu} \leqslant \mathrm{ls} f_{\nu}=-\left(\mathrm{li}-f_{\nu}\right) \leqslant-\left(\mathrm{li}_{\tau}-f_{\nu}\right) .
$$

In each one of the preceding expressions, the first (second resp.) inequality becomes an equality if the collection is $\tau$-equi-1.sc. ( $\tau$-equi-u.sc. resp.).

Let $\bar{C}(X)=S C(X) \cap-S C(X)$ be the space of continuous extended real-valued functions, $\pm e_{\tau}$ the join of the two topologies $e_{\tau}$ and $-e_{\tau}$, and again $p$ the topology of pointwise convergence. In general, $\left(\bar{C}(X), \pm e_{\tau}\right)$ is not compact but as we shall see, its equi-continuous subsets are precompact. A subset $\mathbb{Q} \subset \bar{C}(X)$ is equi-continuous if it is both $\tau$-equi-l.sc. and $\tau$-equi-u.sc.

Proposition 4.7. Suppose that $X$ is separated and locally compact. Then $Q \subset \bar{C}(X)$ is precompact (with respect to $\pm e_{\tau}$ ) if and only if it is equi-continuous.

Proof. If $\mathbb{Q}$ is equi-continuous, it is equi-l.sc. and hence every subset of $Q$ contains a filtered family $\left\{f_{\nu}, \nu \in N\right\}$ such that $\operatorname{lm}_{\tau} f_{\nu}=\operatorname{lm} f_{\nu}$, but by assumption the $\left\{f_{\nu}\right.$, $\nu \in N\}$ are also equi-u.sc. and thus contain a subfamily (a finer filter on $N$ ) such that

$$
\operatorname{lm}_{\tau} f_{\nu}=\operatorname{lm} f_{\nu}=-\left(\operatorname{lm}_{\tau}-f_{\nu}\right)
$$

from it follows that $\mathbb{Q}$ is precompact.

On the other hand, if $Q$ is not equi-continuous, then assume for example, that $\tau$-equi-lower semicontinuity fails. This means that for some collection of functions $\left\{f_{\nu}, \nu \in N\right\}$ and some $x$, we have that

$$
\left(\operatorname{lm}_{\tau} f_{\nu}\right)(x)<\left(\operatorname{li} f_{\nu}\right)(x)=-\left(\operatorname{ls}-f_{\nu}\right)(x) \leqslant-\left(\operatorname{ls}_{\tau}-f_{\nu}\right)(x) .
$$

Hence, there is obviously no subcollection of the $\left\{f_{\nu}\right\}$ whose hypographs converge to $\operatorname{lm}_{\tau} f_{\nu}$, since at $x$ the $-e_{\tau}$-limit inferior of the $\left\{f_{\nu}\right\}$ is strictly larger than $\left(\operatorname{lm}_{\tau} f_{\nu}\right)(x)$. Thus $Q$ cannot be precompact.

Finally, we consider the space $C(X)$ of continuous real-valued functions with the topologies $\pm e_{\tau}, p$ and $\|\cdot\|$, the last one being the sup-norm topology induced by the pseudo-norm defined by $\|f\|=\sup _{x \in X}|f(a)|$. This pseudo-norm induces a topology on $C$. The fundamental system of neighborhoods of an element $f$ is defined by the sets $\{g \in C \mid\|f-g\|<a\}$ with $a>0$. Note that if $X$ is compact, then $\|\cdot\|$ is a norm on $C(X)$ and the topology $\|\cdot\| \subset \pm e_{\tau}$ as can easily be verified. In general, however, these two topologies are not comparable.

THEOREM 4.8. Suppose that $X$ is separated and locally compact and $\mathbb{Q} \subset C(X)$ is equi-continuous and bounded. Then $Q$ is $\pm e_{\tau}$-precompact.

Proof. This follows from the fact that bounded subsets of $S C(X)$ and $-S C(X)$ are $e_{\tau}$ and $-e_{\tau}$-compact, respectively, cf. Corollary 4.4. As in Proposition 4.7 equi-continuity provides the link between the limit functions.

Corollary 4.9 (ARzelÁ-Ascoli). Suppose that $X$ is separated and compact. Then $Q \subset C(X)$ is precompact, with respect to the $\pm e_{\tau}$-topology, and consequently with respect to the $\|\cdot\|$ topology, if and only if $\mathbb{Q}$ is equi-continuous and bounded. 
Sufficiency follows from Theorem 4.8. The necessity of equi-continuity is argued as in Proposition 4.7. Finally, if $Q$ is unbounded, there exist $\left\{f_{\nu}, \nu \in N\right\}$ and $\left\{x_{\nu}\right.$, $\nu \in N\}$ such that $f_{\nu}\left(x_{\nu}\right) \downarrow-\infty$ (or $\uparrow+\infty$ ). The compactness of $X$ implies that the family $\left\{x_{\nu}, \nu \in N\right\}$ admits an accumulation point, say $x$. Then $\left(\operatorname{li}_{\tau} f_{\nu}\right)(x)=-\infty$ (or $-\left(\right.$ li $\left.\left.-f_{\nu}\right)(x)=+\infty\right)$ and hence the $\pm e_{\tau}$-closure of $\mathbb{Q}$ cannot be in $C(X)$ if $\mathbb{Q}$ is unbounded.

Appendix. There is an intimate connection between the semicontinuity properties of multifunctions and the convergence of (filtered) families of sets. The appendix is devoted to clarifying these relations; part of this can be found in one form or another in Choquet [1947-1948] or Kuratowski [1958].

A map $\Gamma$ with domain $Y$ and whose values are subsets of $X$ (possibly the empty set) is called a multifunction. The graph of $\Gamma$ is

$$
\text { grph } \Gamma=\{(y, x) \in Y \times X \mid x \in \Gamma(y)\} .
$$

We recall that the image of $A \subset Y$ is $\Gamma A=\cup_{y \in A} \Gamma(y)$ and the preimage of $B \subset X$ is $\Gamma^{-1} B=\{y \in Y \mid \Gamma(y) \cap B=\varnothing\}$.

A neighborhood base $\mathscr{\Re}\left(y_{0}\right)$ of $y_{0} \in Y$ is a filter base on $Y$. A multifunction $\Gamma$ is said to be upper semicontinuous ( $u . s c$.) at $y_{0}$ whenever

$$
(L s \Gamma)\left(y_{0}\right)=\bigcap_{W \in \mathscr{B}\left(y_{0}\right)} \operatorname{cl} \Gamma W \subset \Gamma\left(y_{0}\right)
$$

or equivalently if to each $x^{0} \notin \Gamma\left(y^{0}\right)$ we can associate neighborhoods $Q$ of $x^{0}$ and $W$ of $y^{0}$ such that $\Gamma W \cap Q=\varnothing$. Note that $\Gamma$ is u.sc. (at every $y$ ) if and only if grph $\Gamma$ is closed.

In the literature one can find a couple of closely connected definitions of upper semicontinuity. A multifunction $\Gamma$ is said to be $K$-u.sc. at $y^{0}$, if to each closed set $F$ disjoint of $\Gamma\left(y_{0}\right)$ there corresponds a neighborhood $W$ of $y_{0}$ such that $\Gamma W \cap F=\varnothing$, or equivalently if to each open set $G$ that includes $\Gamma\left(y_{0}\right)$ there corresponds a neighborhood $W$ of $y_{0}$ such that $\Gamma W \subset G$. If $X$ is regular, then $\Gamma$ closed-valued and $K$-u.sc. at $y_{0}$ implies $\Gamma$-u.sc. at $y_{0}$. If $X$ is compact and $\Gamma$ is closed-valued at $y_{0}$ then both notions coincide.

A multifunction is said to be $C$-u.sc. at $y_{0}$, if to each compact set $K$ disjoint of $\Gamma\left(y_{0}\right)$ there corresponds $V$, a neighborhood of $y_{0}$, such that $\Gamma V \cap K=\varnothing$. Obviously u.sc. implies $C$-u.sc. The converse can be obtained with any one of the following assumptions:

(i) $X$ is locally compact;

(ii) $\Gamma^{-1}$ is $K$-u.sc. at every $x_{0}$ (for example, if $f=Y \rightarrow X$ is a continuous function and $\Gamma=f^{-1}$, then $\Gamma^{-1}$ is $K$-u.sc.);

(iii) $X$ is metrizable, $y_{0}$ has a countable neighborhood base and $\Gamma y_{0}$ is closed, cf. Dolecki [1980].

The proof of the last assertion proceeds as follows: Suppose that $\Gamma$ is not u.sc. at $y_{0}$. Then there exists $x_{0} \notin \Gamma y_{0}$ and neighborhood bases $\left\{Q_{\nu}, \nu=1,2, \ldots\right\}$ of $x_{0}$ and $\left\{W_{\nu}, \nu=1,2, \ldots\right\}$ of $y_{0}$ such that for all $\nu$,

$$
\Gamma y_{0} \cap Q_{\nu} \neq \varnothing
$$


because $\Gamma y_{0}$ is closed, and for all $\nu$

$$
\Gamma W_{\nu} \cap Q_{\nu} \neq \varnothing
$$

because $\Gamma$ is not u.sc. at $y_{0}$. For every $\nu$, pick $x_{\nu} \in \Gamma W_{\nu} \cap Q_{\nu}$. The set $K=$ $\left\{x_{1}, x_{2}, \ldots, x_{0}\right\} \subset X$ is compact (every subsequence converges to $x_{0}$ ) and disjoint of $\Gamma y_{0}$ but meets every $\Gamma W$. This contradicts the $C$-u.sc. of $\Gamma$ at $y_{0}$.

A multifunction is lower semicontinuous (l.sc.) at $y_{0}$ if

$$
\Gamma\left(y_{0}\right) \subset(\operatorname{Li} \Gamma)\left(y_{0}\right)=\bigcap_{V \in \mathscr{B}\left(y_{0}\right)} \mathrm{cl} \Gamma V
$$

where $\ddot{\mathscr{G}}\left(y_{0}\right)$ is the grill associated to the filter base $\mathscr{B}\left(y_{0}\right)$, or equivalently if $\Gamma^{-1} G$ is a neighborhood of $y_{0}$ whenever $G$ is an open set that meets $\Gamma\left(y_{0}\right)$.

For a given set $X$, we denote by $\mathscr{P}(X)$ the power set of $X$, i.e., the hyperspace containing all subsets of $X$, by $\mathscr{F}(X)=\mathscr{F}$ the hyperspace of closed subsets of $X$, and ${ }_{0} \mathscr{F}=\mathscr{F} \backslash\{\varnothing\}$. We now consider the multifunction $\Lambda$ from $\mathscr{P}(X)$ into $X$ defined by $\Lambda Q=Q$. We have that $\Lambda^{-1} \mathbb{Q}=\{Q \mid Q \cap \mathbb{Q} \neq \varnothing\}$ and $\left(\Lambda^{-1} \mathscr{Q}\right)^{c}=\left\{F \mid F \subset \mathbb{Q}^{c}\right\}$.

We restrict $\Lambda$ to $\mathscr{F}$. The sets $\left\{\Lambda^{-1} G, G\right.$ open $\}$ form a subbase for a topology on $0_{0} \mathscr{F}$ (but not for $\mathscr{F}$ ). Similarly, the collection $\left\{\left(\Lambda^{-1} K\right)^{c}, K\right.$ compact $\}$ constitutes a subbase for another topology on $\mathscr{F}$. The supremum of these two topologies yields a topology $\mathcal{T}$ on $\mathcal{F}$. It is the coarsest topology for which $\Lambda$ is both l.sc. and $C$-u.sc. The topology $\mathcal{V}$, the Vietoris topology, on $\mathscr{F}$ has a subbase consisting of the collections $\left\{\Lambda^{-1} G, G\right.$ open $\}$ and $\left\{\left(\Lambda^{-1} F\right)^{c}, F\right.$ closed $\}$. It is the coarsest topology for which the multifunction $\Lambda: \mathscr{F} \rightarrow X$ is 1.sc. and $K$-u.sc.

\section{REFERENCES}

[1979a] H. Attouch, Familles d'opérateurs maximaux monotones et mesurabilité, Ann. Mat. Pura Appl. (4) 120, 35-111.

[1979b] __ Sur la $\Gamma$-convergence, Seminaire Brézis-Lions, Collège de France.

[1981] M. Bernard-Mazure, Equi-s.c.i., Г-convergence et convergence simple, Séminaire d'Analyse Convexe, Montpellier-Perpignan, 1981, Exposé 7.

[1981] G. Buttazzo, Su una definizione generale dei $\Gamma$-limiti, Boll. Un. Mat. Ital. B14, 722-744.

[1947-1948] G. Choquet, Convergences, Ann. Inst. Fourier (Grenoble) 23, 55-112.

[1981] S. Dolecki, Role of lower semicontinuity in optimality theory, Proc. Game Theory and Mathematical Economics (O. Moeschlin and D. Pallaschke, eds.), North-Holland, Amsterdam, pp. 265-274.

[1975] E. De Giorgi and T. Franzoni, Su un tipo di convergenza variazionale, Atti Accad. Naz. Lincei Rend. Cl. Sci. Fis. Mat. Natur. (8) 58, 842-850.

[1958] C. Kuratowski, Topologie, PWN, Warsaw.

[1951] E. Michael, Topologies on spaces of subsets, Trans. Amer. Math. Soc. 71, 151-182.

[1969] U. Mosco, Convergence of convex sets and of solutions to variational inequalities, Adv. in Math. 3 , 510-585.

[1977] G. Salinetti and R. Wets, On the relation between two types of convergence for convex functions, J. Math. Anal. Appl. 60, 211-226.

Institute of Mathematics, Polish Academy of Sciences, Warsaw, Poland

Istituto Calcolo della Probabilita, Universita di Roma, Roma, italia

Department of Mathematics, University of Kentucky, Lexington, Kentucky 40506

Current address (R. J.-B. Wets): IIASA, A-2361 Laxenburg, Austria 